alongside a verbal handover. Doctors on call may not be based at Compton Care during working hours. The document is also used day-to-day to communicate ongoing issues. A review of this document was felt to be required to ensure safe, accurate and efficient transfer of patient information.

Methods Individuals who use the document were surveyed. The effectiveness of communication of the information was evaluated. Views regarding what the document should contain were also gathered. A PDSA cycle approach was adopted: change was driven by survey results and by current accepted practice according to literature and Royal College of Physicians guidance. Patient confidentiality and GDPR regulations were considered when reviewing the document.

Results Of those surveyed 30\% did not fully understand what all parts of the document were communicating. The document was reformatted and a description key was added, subsequently increasing understanding to $100 \%$. A description of the handover document is being included in the induction pack given to new doctors on commencing work. Inclusion of 'ceiling of escalation' was supported by $100 \%$ and therefore added. Sections for 'preferred place of death' and 'discharge information' were added as they had been supported by more than $50 \%$. Inclusion of drop down boxes was felt to be helpful in reducing the time taken to update information.

Conclusions Clear communication of pertinent patient information and patients' wishes will reduce errors and ensure better patient care when making decisions out of hours. Electronic patient software that can be accessed remotely has been introduced recently to Compton Care. This is likely to affect how and what information is handed over; further review will be carried out in approximately one year.

\section{DEVELOPING A SUSTAINABLE MODEL OF SUPPORTIVE AND PALLIATIVE CARE FOR PATIENTS WITH END STAGE HEART FAILURE}

Wendy Sturt, Gillian Horne, Jo Sykes, Tim Chester. Rowcroft Hospice, Torbay and South Devon NHS Foundation Trust

\subsection{6/spcare-2020-PCC.175}

Introduction This 18 month project aimed to enhance the opportunities for patients with end-stage heart failure (HF) and their families to benefit from hospice and specialist palliative care services (SPCS) to facilitate them to plan for, and experience better end of life care.

Method A project team comprising representatives from the HF service, care of older people, primary care, hospital palliative care, patients and families and hospice representatives worked alongside a project lead to design a model of care and pathway for people with end stage HF. A new Supportive and Palliative Care in Heart Failure MDT was established, providing an interdisciplinary forum for identification of those at end of life, and aiding referral to SPCS. A patient information leaflet was designed and printed. Education was delivered to the HF team on palliative care principles, and to the SPCS on management of end stage HF.
Results There is now an established pathway for care of these patients which has improved understanding and communication between the HF and specialist palliative care teams. The MDT meeting is held twice monthly and HF referrals to specialist palliative care have more than doubled (96 patients). A documentation audit of advance care plan (ACP) discussions demonstrates that $64 \%$ patients reviewed at MDT had discussions about their wishes. However, less than half of the patients had entries on the Electronic Palliative Care Coordination System (EPaCCS) with only 6\% having documentation of ACP discussions.

Conclusions Feedback such as 'All care and treatment have been excellent'. (Patient) and 'Much better links between heart failure team and palliative care team with great benefits for patients' (Staff) indicate that this collaborative project has been a positive experience, enabling more patients and families to access hospice and SPCS in their locality. Further work is required to increase use of EPaCCS and sharing of ACP discussions.

\section{THE ROLE OF MEDICAL PROVISION IN HOSPICE DAY SERVICES - A REVIEW}

M Macfarlane, Sophie Taylor, Derek Willis. Severn Hospice

\subsection{6/spcare-2020-PCC.176}

Background Hospice day services are well recognised in palliative care provision. However, there is no standardised model for what structure such services take. For some, medical components are the focus, whereas others are orientated towards normalising life. At Severn Hospice, there has been a transition towards a new 'Living Well' concept: an 8-week assessment programme for patients with more complex palliative care needs. Patients attending this programme are reviewed by a doctor within the first 3 weeks, regardless of need. This has raised questions about whether this is appropriate. A review was undertaken to assess what medical needs patients attending the programme had, to inform discussion on the most appropriate model of medical provision moving forward.

Methods A case notes review was conducted for all new patients attending the 'Living Well' programme between 01/ $06 / 18$ and $31 / 05 / 2019$ to assess what medical input was required.

Results A total of 85 new patients attended the 8 -week programme. Of these, 22 (26\%) had no medical input. 63 patients $(74 \%)$ had at least one medical interaction. Although $74 \%$ of patients were reviewed by a doctor, only 40 patients $(47 \%)$ required any form of medical intervention (medication change, admission to IPU, liaison with GP, or referral to another service). Of these, 50\% (20 patients) required only 1 intervention. There was a small cohort of 14 patients $(16 \%$ of all patients) who required multiple interventions. In the 12 month period, 19 emergencies were encountered, which required rapid medical review.

Conclusions It may not be appropriate to 'medicalise' all patients attending hospice day services if they do not derive any benefit from this interaction. Only a small proportion of patients with complex palliative care needs require specialistlevel medical input. The challenge is identifying who these patients are and how to provide this medical input. 